\title{
Prospects of Artificial Intelligence in Ophthalmic Practice
}

\author{
Shivani Sinha ${ }^{1}$, Abhishek Anand ${ }^{1}$ and Rajvardhan Azad ${ }^{2 *}$ \\ ${ }^{1}$ Regional Institute of Ophthalmology, Indira Gandhi Institute of Medical Sciences, Patna, India \\ ${ }^{2}$ Director, Raj Retina and Eye Care Centre, Patna, India
}

*Corresponding author: Rajvardhan Azad, Raj Retina and Eye Care Centre, Gandhi Murthi, 25, Rd Number 8, North Shastri Nagar,

Patna, Bihar 800023, India

ARTICLE INFO

Received: May 18, 2020

Published: May 28, 2020

Citation: Shivani S, Abhishek A, Rajvardhan A. Prospects of Artificial Intelligence in Ophthalmic Practice. Biomed J Sci \& Tech Res 27(5)-2020. BJSTR. MS.ID.004577.

\begin{abstract}
Artificial intelligence (AI) and machine learning (ML) were part of science fictions a decade before. But with ever evolving technology AI has made its way in our daily lives. AI also has brought paradigm shift in healthcare. Ophthalmology is a lucrative speciality for AI application as lot of imaging modality is being used to make diagnostic and therapeutic decisions. The application of AI in ophthalmology mainly focusses on disease with high incidence like diabetic retinopathy, age related macular degeneration, glaucoma, retinopathy of prematurity and cataract. In this review the current application of AI and its prospects is discussed in the ophthalmic practice.
\end{abstract}

Keywords: Artificial Intelligence; Ophthalmology; Diabetic Retinopathy; Cataract; Deep Learning; Machine Learning

\section{Introduction}

In this technology driven era, where does artificial intelligence (AI) stand in our daily personal and professional lives? The machines haven't taken over yet and may never be able to outsmart their creators but indeed they have seeped their way in our daily lives. Most common examples are voice powered personal assistant "Siri", "Alexa" and "Google assistant"; with "Tesla" rolling out cars with predictive capabilities, self-driving features; "Pandora" with musical DNA and "Nest", the thermostat by Google. AI has become an indispensable tool to better our daily lives. AI has already shown proof of concept in medical fields rooted in diagnostic imaging like radiology, pathology and dermatology. Ophthalmology also relies heavily on imaging like colour fundus photography, optical coherence tomography (OCT) and anterior segment photograph [1]. The advantages of AI in medicine are manifold. In ophthalmology it can assist in clinical practice to detect and learn features from large images, help to reduce diagnostic error. It can also help in providing personalized medicine. It can help us gain innovative scientific insight by recognizing disease specific patterns [2]. AI may also pave way for cost efficient telemedicine screening programs worldwide. The global estimated mean for ophthalmologist density is dismal and was estimated to be 31.7 per million populations [3].
The number of ophthalmologists is growing at half the rate of our population aged over 60 years, and there are 23 countries with less than one ophthalmologist per million population [4]. Various studies have demonstrated successful virtual clinic for various retinal disorders and glaucoma screening $[5,6]$.

\section{Understanding Artificial Intelligence}

What really is AI? Artificial intelligence is the broadest term encompassing machine leaning and deep learning. In simple terms $\mathrm{AI}$ is human like intelligence which a machine or computer acquires through various modalities. The term "artificial intelligence" was first coined by John McCarthy in 1955 [7]. He described it as 'the science and engineering of making intelligent machines. AI comprises of machine machine learning (ML), deep learning (DL), conventional machine learning (CML), natural language processing, computer vision, robotics, reasoning, general intelligence, expert system, automated learning, and scheduling [8].

\section{Machine Learning}

Machine learning (ML) is a subset of AI that enables computers to learn on their own with experience. Arthur Samuel, recognised as pioneer of ML, defined it as 'field of study that gives computers 
the ability to learn without being explicitly programmed and has the ability to automatically learn' [9]. Therefore, machine learning is an ever evolving phenomenon.

\section{Deep Learning}

DL refers to machine learning which employs convolutional based neural networks (CNNs). It involves multiple levels of abstraction to process the input data without the need for manual feature engineering automatically and then the intricate structure is recognised through projection onto a lower dimensional manifold [10]. In conventional machine learning input image is classified from hand engineered features whereas in deep learning the features are both extracted and classified from the input directly allowing for fully end to end learning [11].

\section{AI in Ophthalmic Practice: Where do we stand?}

AI based algorithm has been widely investigated and published in diseases like diabetic retinopathy (DR), age related macular degeneration (AMD), cataract, refractive error, glaucoma, keratoconus and retinopathy of prematurity (ROP).

\section{AI in Retina}

In ophthalmic practice diagnosis in retina is heavily relied on color fundus photography and optical coherence tomography. These diagnostic tools allow non-invasive imaging of retinal vasculature and optic nerve head. Thus, the ample imaging in retina makes it a lucrative area of application of AI.

\section{Diabetic Retinopathy}

According to the International Diabetes Federation approximately 425 million people suffer from diabetes. The prevalence of diabetic retinopathy is approximately $28.5 \%$ in the United States and 18\% in India $[12,13]$. Early detection and prompt treatment remain the mainstay for prevention of visual disability and effectively decreases the burden by $90 \%$. In the current era, ophthalmologists screen the patients for DR but various logistical barrier, lack of trained ophthalmologist and cost of visit may limit the patients from getting the screening done. The other suggested way of addressing these issues are obtaining the colour fundus photo and sending it to ophthalmologist and optometrist to read the image thereby increasing the screening but involves time delay [14]. The limitations can be overcome by automated AI based grading systems. In April 2018, the US Food and Drug Administration (FDA) has approved an AI. AI algorithm, developed by IDx, used with Topcon Fundus camera (Topcon Medical) for DR identification [1522]. Various studies have been outlined in Table 1.

Table 1: Brief summary of various studies.

\begin{tabular}{|c|c|c|c|c|c|c|c|}
\hline DL systems & Year & Test Data Sets & $\begin{array}{l}\text { Number of } \\
\text { Images }\end{array}$ & AUC & Sensitivity & Specifity & Comments \\
\hline $\begin{array}{c}\text { Abràmoff et al. } \\
{[16]}\end{array}$ & 2016 & Messidor 2 & 1748 & 0.98 & 96.8 & 87 & Any DR \\
\hline \multirow{2}{*}{$\begin{array}{c}\text { Gulshan et al. } \\
\text { [17] }\end{array}$} & \multirow{2}{*}{2016} & Messidor 2 & 1748 & 0.99 & 87 & 98.5 & \\
\hline & & Eye PACS 1 & 9963 & 0.991 & 90.3 & 98.1 & Referable DR \\
\hline $\begin{array}{c}\text { Gargeya et al. } \\
{[18]}\end{array}$ & 2017 & Messidor 2 & 1748 & 0.94 & 93 & 87 & Any DR \\
\hline \multirow{2}{*}{ Ting et al. [19] } & 2017 & $\begin{array}{l}\text { SIDRP 2010- } \\
2013\end{array}$ & $\begin{array}{l}\text { Multiple set of } \\
\text { images }\end{array}$ & 0.936 & 90.5 & 91.6 & Referable DR \\
\hline & 2017 & $\begin{array}{l}\text { SIDRP 2010- } \\
2013\end{array}$ & $\begin{array}{l}\text { Multiple set of } \\
\text { images }\end{array}$ & 0.958 & 100 & 91.1 & $\begin{array}{c}\text { Vision } \\
\text { threatening DR }\end{array}$ \\
\hline $\begin{array}{l}\text { Abramoff et al. } \\
{[20]}\end{array}$ & 2018 & & 819 & & 87.2 & 90.7 & Any DR \\
\hline \multirow[t]{2}{*}{$\begin{array}{l}\text { Ramchandran et } \\
\text { al. [21] }\end{array}$} & 2018 & Otago & 485 & 0.901 & 84.6 & 79.7 & Referable DR \\
\hline & & Messidor & 485 & 0.98 & 96 & 90 & \\
\hline \multirow{3}{*}{ Shah et al. [22] } & \multirow{3}{*}{2020} & \multirow{3}{*}{ Messidor } & 1200 & 0.907 & 90.37 & $91.03 \%$ & Any DR \\
\hline & & & 1200 & 0.969 & 94.68 & 97.4 & Referable DR \\
\hline & & & 1200 & 0.923 & 91.67 & 92.92 & $\begin{array}{c}\text { Sight } \\
\text { threatening DR }\end{array}$ \\
\hline
\end{tabular}

IDx-DR: It is the first FDA approved AI algorithm for detection of DR and is paired with non-mydriatic retinal camera (TRCNW400, Topcon). It can be used in the office of non-ophthalmic healthcare practitioners. The captured images are sent to a cloudbased server. The IDx-DR uses a deep learning algorithm to detect retinal findings suggestive of DR. The software provides either of the two results: (1) If more than mild DR detected, refer to an eyecare professional (ECP); (2) If the results are negative for more than mild DR, rescreen in 12 months [23]. The FDA approval was based on a study involving 900 subjects in a primary care setting with automated image analysis of two 45-degree digital image based on optic nerve. The images were compared with the stereo, wide field fundus imaging interpreted by the Wisconsin Fundus Photograph Reading Centre (FPRC). AI was able to make a diagnosis in just 20 second after procurement of retinal images. A new entity minimal DR (mtmDR) was defined [24]. Various AI algorithm 
developed for DR screening like Google AI, EyeArt and IDxDR work on platform based on cloud $[16,17,20,25]$. The AI algorithm by Medios Technologies (Singapore) is the first offline software for DR screening integrated with the smart phone-based fundus camera, the Remidio non-mydriatic (NM) fundus-on-phone (FOP) [26]. The sensitivity and specificity of the AI algorithm in detecting referable DR (RDR) was 93\% (95\% CI 91.3\% to 94.7\%) and 92.5\% (95\% CI $90.8 \%$ to $94.2 \%$ ) in Indian population [27].

\section{AI in AMD}

Approximately 288 million patients have been projected to suffer from AMD by 2040 and $10 \%$ of them will have intermediate AMD or worse $[28,29]$. There is an urgent clinical need to have a robust DL system to cater to the geriatric population to screen them for AMD and to further facilitate evaluation in tertiary care centres.

Ting et al reported a clinically acceptable DL system diagnostic performance in detecting referable AMD [19]. The DL system was trained and tested using 108558 retinal images from 38189 patients bur this being primarily a DR centred study few patients of referable AMD was present. Fovea-centred images without macula segmentation were used in this study. Burlina et al. reported a diagnostic accuracy of between $88.4 \%$ and $91.6 \%$, with an AUC of between 0.94 and 0.96 [30]. In this study macula was presegmented. Grassmann et al reported a sensitivity of $84.2 \%$ in the detection of any AMD [31]. All three abovementioned studies did not have any results for external validation on the individual DL systems. A predictive model also differentiated converting versus non converting eyes with a performance of 0.68 and 0.80 for CNV and GA, respectively [32].

Finally, AI is being studied as a support to therapy decisionmaking in AMD. Prahs et al. looked at AI to support therapy decisions for intravitreal injection and found that the deep CNN was able to correctly predict the need for anti-VEGF therapy in 95\% of cases [33]. Schlegl et al. also designed an MLC using 1200 OCT images with a mean accuracy of 0.94 and an AUROC of 0.92 [34].

\section{AI in Other Retinal Condition}

In 2018 DeepMind applied DL algorithm to OCT and successfully developed a model which automatically segmented tissue layers. The model achieved a diagnostic performance meeting and exceeding those of human expert graders for 50 common retinal conditions. This has paved a way for implementation in a realworld clinical pathway; the rapid access 'virtual' clinics that are now widely used for triaging of macular disease in the UK [5]. In the longer term, the system can be utilised by optometrist too, as OCT has become a common tool, in triaging the patient outside hospitals in community.

\section{AI in Glaucoma}

Glaucoma requires lifelong treatment and monitoring and is the second largest cause of irreversible blindness worldwide primarily affecting the elderly. It is projected that nearly 79.6 million people will have glaucoma by 2020 [35,36]. The first aim is detection of glaucoma based on: classification of visual fields, optic nerve imaging or other clinical data. The second aim is to detect worsening earlier than conventional algorithms available. The last but not the least being able to study risk factors for glaucoma and quality of life based on AI models. Li et al. [37] and Ting et al. [19] trained computer algorithms to detect the glaucoma-like disc. Machine learning methods have been applied to distinguish glaucomatous nerve fibre layer damage from normal scans on wide-angle OCTs [38]. Elze et al. [39] developed an unsupervised computer program to analyse VF that recognises clinically relevant VF loss patterns and assigns a weighting coefficient for each of them and has proven useful in the detection of early VF loss from glaucoma [40]. Computer programs to detect VF progression exist along with sectoral VF analysis. But these approaches are often not aligned with clinical ground truth nor with one another [41,42]. Yousefi et al developed a machine-based algorithm that detected VF progression earlier than these conventional strategies [43]. More machine learning algorithms that provide quantitative information about regional VF progression can be expected in the future.

\section{AI in Cataract}

Table 2: Brief summary of AI studies in cataract.

\begin{tabular}{|c|c|c|c|c|c|}
\hline Authors & Year & Slit Lamp Photograph & $\begin{array}{l}\text { No of Images for } \\
\text { Training }\end{array}$ & $\begin{array}{c}\text { No of Images for } \\
\text { Testing }\end{array}$ & Performance \\
\hline Li et al. [45] & 2009 & Slit lamp photograph & 100 & 5490 & $\begin{array}{c}\text { Success rate of lens structure } \\
\text { detection } 1 / 495 \%\end{array}$ \\
\hline Xu et al. [46] & 2013 & Slit lamp photograph & 100 & 5278 & $\mathrm{MAE}=0.336$ \\
\hline Gao et al. [47] & 2015 & Slit lamp photograph & 100 & 5278 & $\mathrm{MAE}=1 / 40.304$ \\
\hline Wu et al. [48] & 2019 & Slit lamp photograph & 30312 & 7506 & $\begin{array}{l}\text { AUC) of }>99 \% \text { for the capture mode } \\
\text { recognition and cataract detection } \\
\text { phase. } \\
\text { AUC } 0.9915 \text { for evaluation of cataract } \\
\text { severity. } \\
\text { AUC was } 1.00 \text { for referral accuracy for } \\
\text { pediatric cataracts with visual axis } \\
\text { involvement (AUC } 1.00 \text { ). }\end{array}$ \\
\hline Dong et al. [49] & 2017 & $\begin{array}{l}\text { Color fundus } \\
\text { photograph }\end{array}$ & 5495 & 2355 & Accuracy 94.07 \\
\hline
\end{tabular}




\begin{tabular}{|c|c|c|c|c|c|}
\hline Ran et al. [50] & 2018 & $\begin{array}{l}\text { Color fundus } \\
\text { photograph }\end{array}$ & Details not present & Details not present & $\begin{array}{c}\text { AUC } 97.04 \\
\text { Sensitivity } 97.26 \text { Specificity } 96.92\end{array}$ \\
\hline Pratap et al. [51] & 2019 & $\begin{array}{l}\text { Color fundus } \\
\text { photograph }\end{array}$ & 400 & 400 & $\begin{array}{c}\text { Accuracy } 100 \\
\text { For pre trained CNN for feature } \\
\text { extraction }\end{array}$ \\
\hline
\end{tabular}

Cataract is the leading cause of visual impairment worldwide, accounting for 65.2 million cases of vision impairment and blindness globally [44]. These numbers are projected to increase to 70.5 million by 2020 worldwide as the population will age and therefore, they remain an important health concern. Various AI studies have been outlined in Table 2 [45-51]. Wu et al. utilized deep learning using residual neural network (ResNet) to establish a 3-step sequential AI algorithm for the diagnosis and referral of cataracts [48]. First, in the capture mode recognition phase, the AI system would first differentiate slit lamp photographs between mydriatic and nonmydriatic images, and between optical section and diffuse slit-lamp illumination. The second step would involve categorization of image into either normal (ie, no cataract), cataractous, or postoperative IOL. The third step would entail evaluation of type and severity of cataract based on the Lens Opacities Classification System II scale. A decision would be made whether to follow-up or refer the patient for tertiary care would be derived.

The system was validated using 37,638 photographs $(18,819$ eyes) from a Chinese cataract screening program, the AI achieved area under the receiver-operating characteristic curve (AUC) of $>99 \%$ for both steps 1 and 2, AUCs for evaluation of cataract severity (step 3) were most optimal using mydriatic images with optical sections (AUC 0.9915) and least optimal using nonmydriatic images with diffuse illumination (AUC 0.9328), whereas AUCs for referral accuracy were highest for pediatric cataracts with visual axis involvement (AUC 1.00), and lowest for posterior capsular opacification with visual axis involvement (AUC 0.919). This AI algorithm was put on trial as part of a web- based platform, in a pilot study conducted in the Yuexiu district of Guangzhou, China. On comparing with ophthalmologist's final diagnosis, sensitivity and specificity of the algorithm for cataract detection were $92.00 \%$ and $83.85 \%$, respectively. Based on results of pilot study, the authors proposed to switch the first "point- of-care" from ophthalmologists to community-based health care facilities based on AI algorithm. Further AI is being used in IOL power calculation. A new machine learning technique by Gonzalez et al showed that the SD of the prediction error in order of lowest to highest was the new method (designated Karmona) (0.30), Haigis (0.36), Holladay 2 (0.38), Barrett Universal II (0.38), and Hill-RBF v2.0 (0.40). Using the Karmona method, $90.38 \%$ and $100 \%$ of eyes were within \pm 0.50 and \pm 1.00 D respectively [52].

\section{AI for Corneal Diseases}

Table 3: Various studies in corneal ectasias.

\begin{tabular}{|c|c|c|c|c|c|}
\hline Authors & Year & Purpose & AUC & Sensitivity & Specifity \\
\hline Souza et al. [56] & 2010 & $\begin{array}{c}\text { Differentiate normal, } \\
\text { astigmatic, photorefractive } \\
\text { keratectomy and } \\
\text { keratoconus corneas }\end{array}$ & 0.98-0.99 & $0.98-1.00$ & $0.98-1.00$ \\
\hline Hidalgo et al. [57] & 2017 & $\begin{array}{l}\text { Differentiate normal, } \\
\text { keratoconus and sub-clinical } \\
\text { corneal ectasia }\end{array}$ & $\begin{array}{c}\text { keratoconus vs normal } \\
0.998 ; \text { subclinical vs } \\
\text { normal } 0.922\end{array}$ & $\begin{array}{l}\text { keratoconus vs } \\
\text { normal:0.991 subclinical } \\
\text { vs normal:0.791 }\end{array}$ & $\begin{array}{c}\text { keratoconus vs } \\
\text { normal:0.998 subclinical } \\
\text { vs normal:0.979 }\end{array}$ \\
\hline Arbelaez et al. [58] & 2012 & $\begin{array}{l}\text { Differentiate normal, } \\
\text { keratoconus and sub-clinical } \\
\text { corneal ectasia }\end{array}$ & Not reported & $\begin{array}{l}\text { keratoconus vs } \\
\text { normal:0.950 subclinical } \\
\text { vs normal:0.920 }\end{array}$ & $\begin{array}{c}\text { keratoconus vs } \\
\text { normal:0.993 subclinical } \\
\text { vs normal:0.977 }\end{array}$ \\
\hline Kovacs et al. [59] & 2016 & $\begin{array}{l}\text { Differentiate normal, } \\
\text { keratoconus and sub- } \\
\text { clinical corneal ectasia using } \\
\text { bilateral data) }\end{array}$ & $\begin{array}{c}\text { keratoconus versus } \\
\text { normal } 0.99 \text { subclinical } \\
\text { versus normal:0.96 }\end{array}$ & $\begin{array}{c}\text { keratoconus vs } \\
\text { normal:1.00subclinical vs } \\
\text { normal: } 0.90\end{array}$ & $\begin{array}{c}\text { keratoconus vs } \\
\text { normal:0.95 subclinical vs } \\
\text { normal: } 0.90\end{array}$ \\
\hline $\begin{array}{l}\text { Ambrosio et al. } \\
\text { [60] }\end{array}$ & 2017 & $\begin{array}{c}\text { Differentiate normal, } \\
\text { keratoconus and sub- } \\
\text { clinical corneal ectasia using } \\
\text { tomographic biomechanical } \\
\text { index) }\end{array}$ & 0.996 & $\begin{array}{c}\text { Detecting ectasia vs } \\
\text { normal: } 1.00 \text { subclinical vs } \\
\text { normal:0.904 }\end{array}$ & $\begin{array}{c}\text { Detecting ectasia vs } \\
\text { normal: } 1.00 \text { subclinical vs } \\
\text { normal:0.960 }\end{array}$ \\
\hline
\end{tabular}

Keratoconus being a progressive disease has attracted the attention of AI developers to detect it at the earliest. This can help in better preservation of vision in these patients. Also, with an increase in number of refractive surgeries the number of iatrogenic keratectasias is on the rise because of possible biomechanics failure which we were unable to pick before surgery. So to identify these susceptible corneas various AI models have been developed (Table 1). In diabetic sensorimotor polyneuropathy with introduction of neural network and random forest models, the nerve can be fully segmented, and morphology can be studied hence allowing the development of an objective and precise method to early characterise the disease $[53,54]$. AI models can predict early 
graft dislocation based on larger residual interface fluid following refractive surgery [55]. Identifying subclinical corneal ectasia is challenging but the topography and tomography provides data to the ophthalmologist for every cornea, but still subclinical keratoconus remains a diagnostic challenge. Often a decision is often made by individual ophthalmologist's subjective interpretation of patterns or empiric cut-off values, which vary from machine to machine thus is unreliable and time consuming. In this scenario AI could provide an alternative solution to recognising those patients most at risk and diagnosing subclinical ectasias (Table 3) [56-60].

\section{AI in Paediatric Ophthalmology}

\section{Retinopathy of Prematurity (ROP)}

It is intuitive to expect that AI in ROP has a colossal potential. ROP compared to DR is relatively limited by retinal findings but the complexity lies in the urgency of intervention in treatable ROP and in the management of systemic comorbidity in a neonate compared to adults with DR. Several semi-automated AI tools like Vessel Finder [61], VesselMap [62], ROPtool [63], Retinal Image multiScale Analysis [64-66] , Computer- Aided Image Analysis of the Retina $[67,68]$, and IVAN $[67,69]$ have been studied. These CNN models accurately assessed retinal fundus image quality in ROP in a manner comparable with the experts. The i-ROP deep learning [70] and DeepROP system [71] have been found to accurately identify diagnostic categories and overall disease severity in an automated fashion. This iROP DL system has only been trained on posterior pole vascular morphology. The data provides a proof to concept that a deep learning system can be used in an automated fashion to diagnose ROP. These tools when incorporated in successful tele ROP programs can be revolutionary as it will help in early and rapid triage of infants needing treatment even in rural areas. AI in ROP is going to have a tremendous impact in a country like India where preterm births have crossed 3.5 million mark annually more than any other country. Estimated 215 specialist that is even less than $1 \%$ of the ophthalmologists are engaged in ROP services directly. $\mathrm{AI}$ seems to provide us with the solutions and mend road ahead for optimal ROP care

\section{Eye as a Window}

For long philosophers has defined the eye as a window to the soul before scientists addressed this cliché to prove its scientific basis and clinical relevance. Recent work has applied DNN on retinal OCT and fundus images for assessing age, gender, systolic blood pressure, smoking status, haemoglobin A1c, and likelihood of having a major adverse cardiac event. The AUC for gender was found to be 0.97 while the others were in the range of 0.70 . This suggests that through further advancements many more information can be gathered by these images using AI based algorithms [72]. AI has widespread application in ophthalmological practice. AI still is in its stage of infancy. There are three main limitation of AI which needs consideration [73]. Usually, the studies use homogenous population data as training data sets. This leads to their questionable generalisation in diverse population and different ethnicity. The second pitfall being the "black box" nature of the AI where the physician is not able to understand the algorithm being used. This leads to trust issues with the AI platforms. The third being the power calculation done for an independent data sets used in these studies. This brings a questionable calibration of the algorithm being used in these AI platforms. In addition, the medico-legal aspects and the regulatory approvals vary in different countries and need to be addressed.

Nonetheless, with development of the Eye Grader clinician interface a new era has ushered where an immediate grading report for clinicians are generated in an integrated and automated fashion that can function both online and offline. Eye Grader has been designed to grade for four common blinding eye diseases including referable DR, suspect glaucoma, wet AMD, and cataract. The feasibility and acceptability of the Eye Grader system has been pilot-tested in real-world settings in Australia with great success and commendable patient satisfaction [74]. These AI systems will help bridge the significant patient doctor ratio gap. Even the patient in remote areas would be able to access the specialised healthcare system. The AI platforms also will enable health care providers like physicians and endocrinologist, who are first point contact in systemic diseases, to perform screening procedures in their patient at the earliest. Even non eye health care professional would be able to actively involved in the screening and diagnosis in the early stage of disease. This in turn will lead to timely diagnosis and treatment thereby bringing down the burden of visual impairment and blindness.

\section{Conclusion}

$\mathrm{AI}$ is the next big leap in ophthalmology. With combined clinical skill of ophthalmologists and AI platforms the medicine field is all set for the next big revolution. The long lived dream of medical care for everyone, at every doorstep has been an ever alluding dream since long but with each passing day we are inching closer to get this dream fulfilled with mankind's one of the great invention "AI".

\section{Acknowledgement}

None.

\section{Conflict of Interest}

No conflict of interest.

\section{References}

1. Jiang F, Jiang Y, Zhi H, Dong Y, Li H, et al. (2017) Artificial intelligence in healthcare: past, present and future. Stroke and vascular neurology 2(4): 230-243.

2. Schmidt-Erfurth U, Sadeghipour A, Gerendas BS, Waldstein SM, Bogunović H (2018) Artificial intelligence in retina. Prog Ret Eye Res 67: 1-29.

3. Resnikoff S, Lansingh VC, Washburn L, Felch W, Gauthier TM, et al. (2019) Estimated number of ophthalmologists worldwide (International Council of Ophthalmology update): will we meet the needs? $\mathrm{Br} \mathrm{J}$ Ophthalmol pp. 314336. 
4. Resnikoff S, Felch W, Gauthier TM, Spivey B (2012) The number of ophthalmologists in practice and training worldwide: a growing gap despite more than 200000 practitioners. Br J Ophthalmol 96: 783-787.

5. Kortuem K, Fasler K, Charnley A, Hussain Khambati, Sandro Fasolo, et al. (2018) Implementation of medical retina virtual clinics in a tertiary eye care referral centre. Br J Ophthalmol 102: 1391-1395.

6. Kotecha A, Brookes J, Foster PJ (2017) A technician-delivered 'virtual clinic'for triaging low-risk glaucoma referrals. Eye 31: 899-905.

7. Mc Carthy J, Minsky ML, Rochester N, Claude E Shannon (2006) A proposal for the Dartmouth Summer Research Project on Artificial Intelligence, August 31, 1955. AI Magazine pp. 2712-2714.

8. Balyen L, Peto T (2019) Promising artificial intelligence-machine learning-deep learning algorithms in ophthalmology. Asia Pac J Ophthalmol 8: 264-272.

9. Samuel AL (1988) Some studies in machine learning using the game of checkers. I. In: Levy DNL, editor. Computer Games I. Springer, New York, USA, pp. 335-365.

10. LeCun Y, Bengio Y, Hinton G (2015) Deep learning. Nature 521(7553): 436-444.

11. Schmidt-Erfurth U, Sadeghipour, A, Gerendas BS, Waldstein SM Bogunović H (2018). Artificial intelligence in retina. Prog Ret Eye Res 67: 1-29.

12. Zhang X, Saaddine JB, Chou CF, Mary Frances Cotch, Yiling J Cheng, et al (2010) Prevalence of diabetic retinopathy in the United States, 20052008. JAMA 304: 649-656.

13. Raman R, Rani PK, Reddi Rachepalle S, Perumal Gnanamoorthy, Satagopan Uthra, et al. (2009) Prevalence of diabetic retinopathy in India: Sankara Nethralaya Diabetic Retinopathy Epidemiology and Molecular Genetics Study report 2. Ophthalmology 116: 311-318.

14. Taylor CR, Merin LM, Salunga AM, Hepworth JT, Crutcher TD, et al. (2007) Improving diabetic retinopathy screening ratios using telemedicinebased digital retinal imaging technology: The Vine Hill study. Diabetes Care 30: 574-578.

15. US Food and Drug Administration. FDA permits marketing of artificial intelligence-based device to detect certain diabetes-related eye problems

16. Abràmoff MD, Lou Y, Erginay A, Clarida W, Amelon R, et al. (2016) Improved automated detection of diabetic retinopathy on a publicly available dataset through integration of deep learning. Invest Ophthalmol Vis Sci. 57: 5200-5206.

17. Gulshan V, Peng L, Coram M, Stumpe MC, Wu D, et al. (2016) Development and validation of a deep learning algorithm for detection of diabetic retinopathy in retinal fundus photographs. Jama 316: 2402-2410.

18. Gargeya R, Leng T (2017) Automated identification of diabetic retinopathy using deep learning. Ophthalmology 124: 962-969.

19. Ting DSW, Cheung CY, Lim G, Tan GSW, Quang ND, et al. (2017) Development and validation of a deep learning system for diabetic retinopathy and related eye diseases using retinal images from multiethnic populations with diabetes. JAMA 318: 2211-2223.

20. Abramoff MD, Lavin PT, Birch M, Shah N, Folk JC (2018) Pivotal trial of an autonomous AI-based diagnostic system for detection of diabetic retinopathy in primary care offices. NPJ Digit Med 1: 39.

21. Ramachandran N, Hong SC, Sime MJ, Wilson GA (2018) Diabetic retinopathy screening using deep neural network. Clin Exp Ophthalmol 46: 412-416.

22. Shah P, Mishra DK, Shanmugam MP, Doshi B, Jayaraj H, et al. (2020) Validation of Deep Convolutional Neural Network-based algorithm for detection of diabetic retinopathy-Artificial intelligence versus clinician for screening. Indian J Ophthalmol 68: 398-405.
23. Abramoff M (2018) Artificial intelligence for automated detection of diabetic retinopathy in primary care. Paper presented at: Macular Society; February 22, 2018; Beverly Hills, CA.

24. Pros and Cons of Using an AI-Based Diagnosis for Diabetic Retinopathy: Page 4 of 5 N.d. Optometry Times.

25. Bhaskaranand M, Ramachandra C, Bhat S, Jorge Cuadros, Muneeswar G Nittala, et al. (2019) The value of automated diabetic retinopathy screening with the EyeArt system: a study of more than 100,000 consecutive encounters from people with diabetes. Diabetes Technol Ther 21: 635-643.

26. Rajalakshmi R, Arulmalar S, Usha M, Vijayaraghavan Prathiba, Khaji Syed Kareemuddin, et al. (2015) Validation of smartphone based retinal photography for diabetic retinopathy screening. PLoS One 10: e0138285.

27. Sosale B, Aravind SR, Murthy H, Narayana S, Sharma U, et al. (2020) Simple, Mobile-based Artificial Intelligence Algorithm in the detection of Diabetic Retinopathy (SMART) study. BMJ Open Diabetes Research Care 8: e000892.

28. Chew EY, Clemons TE, Agrón E, Lenore J Launer , Francine Grodstein et al. (2015) Effect of omega-3 fatty acids, lutein/zeaxanthin, or other nutrient supplementation on cognitive function: the areds 2 randomized clinical trial. JAMA 314: 791-801.

29. Wong WL, Su X, Li X, Chui Ming G Cheung, Ronald Klein, et al. (2014) Global prevalence of age-related macular degeneration and disease burden projection for 2020 and 2040: a systematic review and metaanalysis. Lancet Glob Health 2: e106-116.

30. Burlina PM, Joshi N, Pekala M, Katia D Pacheco, David E Freund, et al. (2017) Automated grading of age-related macular degeneration from color fundus images using deep convolutional neural networks. JAMA Ophthalmol 135: 1170-1176.

31. Grassmann F, Mengelkamp J, Brandl C, Sebastian Harsch , Martina E Zimmermann, et al. (2018) A deep learning algorithm for prediction of age-related eye disease study severity scale for age-related macular degeneration from color fundus photography. Ophthalmology 125: 1410-1420.

32. Schmidt-Erfurth U, Waldstein SM, Klimscha S, Sadeghipour A, Hu X, et al. (2018) Prediction of individual disease conversion in early AMD using artificial intelligence. Invest Ophthalmol Vis Sci 59: 3199-3208.

33. Prahs P, Märker D, Mayer C, Helbig H (2018) Deep learning to support therapy decisions for intravitreal injections. Der Ophthalmologe: Zeitschrift der Deutschen Ophthalmologischen Gesellschaft 115: 722727.

34. Schlegl T, Waldstein SM, Bogunovic H, Franz Endstraßer 3, Amir Sadeghipour, et al. (2018) Fully Automated Detection and Quantification of Macular Fluid in OCT Using Deep Learning. Ophthalmology 125: 54958.

35. Resnikoff S, Pascolini D, Etya'ale D, Ivo Kocur, Ramachandra Pararajasegaram, et al. (2004) Global data on visual impairment in the year 2002. Bull World Health Organ 82: 844-851.

36. Quigley HA, Broman AT (2006) The number of people with glaucoma worldwide in 2010 and 2020. Br J Ophthalmol 90: 262-267.

37. Li Z, He Y, Keel S, Stuart Keel, Wei Meng, et al. (2018) Efficacy of a deep learning system for detecting glaucomatous optic neuropathy based on color fundus photographs. Ophthalmology 125: 1199-206.

38. Christopher M, Belghith A, Weinreb RN, Christopher Bowd, Michael H Goldbaum, et al. (2018) Retinal nerve fiber layer features identified by unsupervised machine learning on optical coherence tomography scans predict glaucoma progression. Invest Ophthalmol Vis Sci 59: 2748-2756.

39. Elze T, Pasquale LR, Shen LQ, Teresa C Chen, Janey L Wiggs, et al. (2015) Patterns of functional vision loss in glaucoma determined with archetypal analysis. J R Soc Interface 12: 20141118 
40. Wang M, Pasquale LR, Shen LQ Michael V Boland, Sarah R Wellik, et al. (2018) Reversal of glaucoma hemifield test results and visual field features in glaucoma. Ophthalmology 125: 352-360.

41. Tanna AP, Bandi JR, Budenz DL, William J Feuer, Robert M Feldman, et al. (2011) Interobserver agreement and intraobserver reproducibility of the subjective determination of glaucomatous visual field progression. Ophthalmology 118: 60-65.

42. Viswanathan AC, Crabb DP, Mc Naught AI, e M C Westcott, D Kamal, et al. (2003) Interobserver agreement on visual field progression in glaucoma: a comparison of methods. Br J Ophthalmol 87: 726-730.

43. Yousefi S, Kiwaki T, Zheng Y, Hiroki Sugiura, Ryo Asaoka, et al. (2018) Detection of longitudinal visual field progression in glaucoma using machine learning. Am J Ophthalmol 193: 71-79.

44. Flaxman SR, Bourne RRA, Resnikoff S, Peter Ackland, Tasanee Braithwaite, et al. (2017) Global causes of blindness and distance vision impairment 1990-2020: a systematic review and meta- analysis. Lancet Global Health 5: e1221--e1234.

45. Li H, Lim JH, Liu J, Damon Wing Kee Wong, Ngan Meng Tan, et al. (2009) An automatic diagnosis system of nuclear cataract using slit-lamp images. Conf Proc IEEE Eng Med Biol Soc 2009: 3693-3696.

46. Xu Y, Gao X, Lin S, Damon Wing Kee Wong, Jiang Liu, et al. (2013) Automatic Grading of Nuclear Cataracts from Slit-Lamp Lens Images Using Group Sparsity Regression. Berlin, Heidelberg: Springer Berlin Heidelberg pp. 468-475.

47. Gao X, Lin S, Wong TY (2015) Automatic feature learning to grade nuclear cataracts based on deep learning. IEEE Trans Biomed Eng 62: 2693- 2701.

48. Wu X, Huang Y, Liu Z, Weiyi Lai, Erping Long, et al. (2019) Universal artificial intelligence platform for collaborative management of cataracts. Br J Ophthalmol 103: 1553-1560.

49. Dong Y, Zhang Q, Qiao Z, Yang J (2017) Classification of cataract fundus image based on deep learning. 2017 IEEE International Conference on Imaging Systems and Techniques (IST): 1-5.

50. Ran J, Niu K, He Z, Zhang H, Song H (2018) Cataract Detection and Grading Based on Combination of Deep Convolutional Neural Network and Random Forests. 2018 International Conference on Network Infrastructure and Digital Content (IC-NIDC): 155-159.

51. Pratap T, Kokil P (2019) Computer-aided diagnosis of cataract using deep transfer learning. Biomed Signal Process Control 53: 101533.

52. González DC, Bautista CP (2020) Accuracy of a new intraocular lens power calculation method based on artificial intelligence. Eye 28: 1-6.

53. Al-Fahdawi S, Rami Qahwaji , Alaa S Al-Waisy, Stanley Ipson, Rayaz A Malik, et al. (2016) A fully automatic nerve segmentation and morphometric parameter quantification system for early diagnosis of diabetic neuropathy in corneal images. Comput Methods Programs Biomed 135: 151-166.

54. Chen X, Graham J, Dabbah MA, Petropoulos IN, Tavakoli M, et al. (2017) An automatic tool for quantification of nerve fibers in corneal confocal microscopy images. IEEE Trans Biomed Eng 64: 786-794.

55. Treder M, Jost Lennart Lauermann, Maged Alnawaiseh, Nicole Eter (2019) Using deep learning in automated detection of graft detachment in Descemet membrane endothelial keratoplasty: a pilot study. Cornea 38: $157-161$

56. Souza MB, Medeiros FW, Souza DB, Garcia R, Alves MR (2010) Evaluation of machine learning classifiers in keratoconus detection from orbscan II examinations. Clinics 65: 1223-1228.

57. Ruiz Hidalgo I, Rozema JJ, Saad A, Pablo Rodriguez, Nadia Zakaria, et al. (2017) Validation of an Objective Keratoconus Detection System Implemented in a Scheimpflug Tomographer and Comparison with Other Methods. Cornea 36: 689-695.
58. Arbelaez MC, Versaci F, Vestri G, Barboni P, Savini G (2012) Use of a support vector machine for keratoconus and subclinical keratoconus detection by topographic and tomographic data. Ophthalmology 119: 2231-2238.

59. Kovacs I, Mihaltz K, Kranitz K, Éva Juhász, A Takács, et al. (2016) Accuracy of machine learning classifiers using bilateral data from Schiempflug camera for identifying eyes with preclinical signs of keratoconus. J Cataract Refract Surg 42: 275-283.

60.Ambrósio R Jr, Lopes BT, Faria-Correia F, Marcella Q Salomão, Jens Bühren, et al. (2017) Integration of Scheimpflug- Based Corneal Tomography and Biomechanical Assessments for Enhancing Ectasia Detection. J Refract Surg 33: 434-443.

61. Heneghan C, Flynn J, O’Keefe M, Cahill M (2002) Characterization of changes in blood vessel width and tortuosity in retinopathy of prematurity using image analysis. Med Image Anal 6:407-429.

62. Rabinowitz MP, Grunwald JE, Karp KA, Gui-Shuang Ying, Monte D Mills (2007) Progression to severe retinopathy predicted by retinal vessel diameter between 31 and 34 weeks of postconception age. Arch Ophthalmol 125: 1495-1500.

63. Gelman R, Jiang L, Du YE, M Elena Martinez-Perez, John T Flynn, et al. (2007) Plus disease in retinopathy of prematurity: pilot study of computer-based and expert diagnosis. J Am Assoc Pediatr Ophthalmol Strabismus 11: 532-540.

64. Wallace DK, Zhao Z, Freedman SF (2007) A pilot study using 'ROPtool' to quantify plus disease in retinopathy of prematurity. J Am Assoc Pediatr Ophthalmol Strabismus 11: 381-387.

65. Swanson C, Cocker KD, Parker KH, M J Moseley, A R Fielder (2003) Semiautomated computer analysis of vessel growth in preterm infants without and with ROP. Br J Ophthalmol 87: 1474-1477.

66. Gelman R, Martinez-Perez ME, Vanderveen DK, Anne Moskowitz, Anne B Fulton (2005) Diagnosis of plus disease in retinopathy of prematurity using retinal image multiscale analysis. Invest Opthalmol Vis Sci 46: 4734-4738.

67. Shah DN, Wilson CM, Ying GS, Karen A Karp, Alistair R Fielder, et al (2009) Semiautomated digital image analysis of posterior pole vessels in retinopathy of prematurity. J Am Assoc Pediatr Ophthalmol Strabismus 13: 504-506.

68. Wilson CM, Cocker KD, Moseley MJ, Carl Paterson, Simon T Clay, et al. (2008) Computerized analysis of retinal vessel width and tortuosity in premature infants. Invest Ophthalmol Vis Sci 49: 3577-3585.

69. Sherry LM, Jin Wang J, Rochtchina E, TienY Wong, Ronald Klein, et al. (2002) Reliability of computer-assisted retinal vessel measurement in a population. Clin Exp Ophthalmol 30: 179-182.

70. Brown JM, Campbell JP, Beers A, Ken Chang, Susan Ostmo, et al. (2018) Automated diagnosis of plus disease in retinopathy of prematurity using deep convolutional neural networks. JAMA Ophthalmol 136: 803-810.

71. Wang J, Ju R, Chen Y, Lei Zhang, Junjie Hu, et al. (2018) Automated retinopathy of prematurity screening using deep neural networks. EBioMedicine 35: 361-368.

72. Poplin R, Avinash V Varadarajan, Katy Blumer, Yun Liu, Michael V Mc Connell, et al. (2018) Prediction of cardiovascular risk factors from retinal fundus photographs via deep learning. Nat Biomed Eng 2: 158164.

73. Ting DS, Pasquale LR, Peng L, Campbell JP, Lee AY, et al. (2019). Artificial intelligence and deep learning in ophthalmology. Br J Ophthalmol 103: 167-175.

74. Tan Z, Scheetz J, He M (2019) Artificial Intelligence in Ophthalmology: Accuracy, Challenges, and Clinical Application. Asia Pac J Ophthalmol (Phila) 8: 197-199. 
ISSN: 2574-1241

DOI: 10.26717/BJSTR.2020.27.004577

Rajvardhan Azad. Biomed J Sci \& Tech Res

(C) 9 This work is licensed under Creative

Submission Link: https://biomedres.us/submit-manuscript.php

$\begin{array}{ll}\text { BIOMEDICAL } & \text { Assets of Publishing with us } \\ \text { RESEARCHES } & \text { - Global archiving of articles } \\ \text { - Immediate, unrestricted online access } & \text { - Rigorous Peer Review Process } \\ & \text { - Authors Retain Copyrights } \\ & \end{array}$

